\title{
Incertidumbre en los proyectos de investigación y desarrollo (I+D) Un estudio de la literatura*
}

\author{
Ángel Samaniego \\ Alcántar \\ Instituto de Estudios Superio- \\ res de Occidente. \\ asamanie@iteso.mx
}

*El autor agradece al Instituto Tecnológico y de Estudios Superiores de Occidente por el financiamiento a esta investigación, a Dídac Ramírez de la Universidad de Barcelona y a los árbitros anónimos por los comentarios y aportaciones a este documento; es responsabilidad del autor cualquier error derivado del mismo.

\section{Resumen}

La incertidumbre que existe al invertir en proyectos de investigación y desarrollo (I+D) origina que éstos se realicen en diferentes etapas. La decisión de continuar o abandonar un proyecto se toma con base en las diferentes señales que se van presentando en él (término de una etapa de la investigación, demanda del producto, precios de materias primas en el tiempo, reacción de competidores, entre otras). De igual forma, la dificultad de valorar y seleccionar estos proyectos se centra en los riesgos en las diferentes etapas de su desarrollo, por lo que se puede llegar a sobrevaluar o subvalorar el proyecto. Debido a lo anterior, este trabajo tiene la finalidad de presentar los avances que hay sobre el tema, intentando identificar problemáticas y metodologías estudiadas en los últimos años. Las bases metodológicas que se encontraron se ubican dentro del análisis multicriterio, teoría de opciones, la teoría de la posibilidad y la teoría de los conjuntos borrosos.

Palabras clave: proyectos de inversión, incertidumbre, conjuntos borrosos, opciones reales, portafolios de inversión.

JEL Classification: O22, D81. 


\title{
Uncertainty in $R \& D$ projects: literature review
}

\begin{abstract}
Due to the high level of uncertainty of research and development (R\&D) investment projects, such projects are undertaken by stages. At different points in time those projects are reviewed as options to continue or abandon them arise. This decision depends upon signals provided by the projects themselves during their investment time-frame (conclusion of a research stage, product demand, raw materials price, competition, among others). Given this high uncertainty about risks in $\mathrm{R} \& \mathrm{D}$ projects it is difficult to value them, which may lead to either overvalue or undervalue them. This article attempts to present recent advances on the subject by identifying problems and methodologies under study in recent years. In general, currently used methodologies are categorized within multi-criteria analyses, options theory, and the theory of possibilities from a fuzzy-sets perspective.
\end{abstract}

Keywords: investment project, uncertainty, fuzzy sets, real option, investment portfolio.

\section{Introducción}

Los diferentes estudios sobre las relaciones entre innovación y el crecimiento económico han puesto de manifiesto cómo la innovación afecta al crecimiento (Fagerberg, Mowery y Nelson, 2005). La innovación es una idea relacionada con algo comercialmente aceptado (Escorsa y Maspons, 2001). En su forma básica puede ser: innovación de producto (mercancías o servicios nuevos); innovación de procesos, se cambia la manera de producir un bien a una forma que aumente su rentabilidad; e innovación organizacional, habilidad en la utilización de los recursos de una organización para crear una idea que represente una mejor posición en sus objetivos. Estos tres tipos de innovación pueden estar relacionados en la economía; por ejemplo, invertir en un proyecto de innovación organizacional nos puede llevar a una mejora en la utilización de los recursos de la organización, y si este proyecto se demanda fuera de la organización (innovación de un producto) mejora los procesos de la empresa que demandó el producto (innovación de procesos). Lo anterior nos lleva a pensar que la inversión en proyectos de investigación y desarrollo (I+D) es un medio por el cual la innovación se extiende por la economía.

Los aumentos en la "eficacia productiva", a través de la innovación, introduce nuevos y mejores métodos de producción; las innovaciones con éxito aumentarán eventualmente el nivel y el ritmo de crecimiento de la productividad en el largo 
plazo; es decir, aumentos de la "eficacia dinámica." Una manera de aumentar esta "eficacia" es fortalecer la capacidad innovadora de las empresas. Lo anterior es resultado de seleccionar e invertir eficientemente en proyectos de I+D. Un proyecto de investigación utiliza el método científico con el objetivo de recabar todo tipo de información para la formulación y contrastación de hipótesis, las cuales se seleccionan junto con las teorías para que sean empíricamente refutables; además, se someten constantemente al riesgo de la refutación empírica y se valoran de acuerdo con los resultados de esa contrastación. Si hay éxito, se obtiene conocimiento y se analiza la posibilidad en convertir este conocimiento en innovación para, posteriormente, desarrollarlo.

Los diferentes métodos de selección de proyectos de $\mathrm{I}+\mathrm{D}$ caen en las siguientes categorías (Henriksen y Traynos, 1999)2:

- Programación matemática y optimización de portafolios: programación lineal, programación no lineal, programación dinámica y optimización de portafolios.

- Modelos económicos: valor presente, retorno de la inversión, análisis de costo-beneficio y la teoría de opciones.

- Análisis de las decisiones: teoría de la utilidad multi-atributo, árboles de decisión, análisis de riesgo, proceso jerárquico analítico (analytic hierarchy process, AHP) y scoring.

- Métodos iterativos: Delphi, Q-sort, behaviour decisión aids y modelado de la jerarquía descentralizada.

- Inteligencia artificial: sistemas expertos y la teoría de conjuntos borrosos.

Ante la diversidad y complejidad de los datos que presenta el proyecto, y su relación en un contexto en continuo movimiento, dificulta la transformación de estos datos en un conocimiento valioso para la toma de decisiones.

\footnotetext{
${ }^{1}$ La eficacia dinámica ocurre en un cierto plazo en el sentido que un mercado debe resolver nuestras necesidades y deseos que cambian mientras progresa el tiempo.

${ }^{2}$ Presentan además una lista de la literatura de la mayoría de los métodos anteriores relacionados con la selección de proyectos de I+D. Johnson, Neave y Pazderka (2002) y Dapena (2003) comentan sobre los métodos tradicionales para la valoración de proyectos de inversión ( $q$ de Tobin, valor presente, modelo de Gordon, Modigliani-Miller, entre otros).
} 
La incertidumbre provoca a la organización a controlar todo con cierta exactitud. Este miedo al fracaso puede alejar a los proyectos de éxito. Este trabajo pretende encontrar las problemáticas y metodologías que se están estudiando sobre la selección y valoración de proyectos de I+D con el fin de clasificarlas y ordenarlas para obtener una relación entre conceptos y metodologías que ayuden a identificar problemas, así como las propuestas metodológicas para su solución; cabe aclarar que en este documento no se explican estas metodologías ni se contrastan.

Esta investigación se conforma de las siguientes partes: en la sección dos se introduce a la valoración de proyectos de inversión; en la sección tres se explica el porqué de la utilización de la teoría de conjuntos borrosos; la sección cuatro resume las publicaciones que utilizan la teoría de conjuntos borrosos en la selección de proyectos de I+D; y en la sección cinco se presentan los resultados del estudio de la literatura, así como se exponen posibles líneas de investigación.

\section{Valoración de proyectos de inversión}

La toma de decisiones sobre invertir o no en un proyecto de inversión lleva a crearnos una expectativa de beneficio futuro. Para encontrar este beneficio de invertir en un momento dado - es posible que el costo de esperar lleve a la aceptación (Dixit y Pindyck, 1994) — se requiere saber las fuentes de valor ${ }^{3}$, de riesgo, costo y el comportamiento del proyecto frente a otros competidores y/o proyectos (consecuencias de las acciones tomadas). Al momento de tomar decisiones la información es asimétrica ${ }^{4}$ y está relacionada una con otra; por ejemplo, no se puede hablar de valor sin tomar en cuenta el riesgo.

Según el método elegido, se puede incorporar en la valoración las diferentes fuentes de valor, riesgo, incertidumbre, costos y el comportamiento del proyecto durante su vida. Conocer bien estas fuentes y los alcances del método elegido contribuirá a mejorar el desempeño en la toma de decisiones en las diferentes etapas de madurez ${ }^{5}$ del proyecto. Cabe aclarar que durante estas etapas se van presentando decisiones de la forma de una opción call $^{6}$, donde el inversionista tiene el derecho,

\footnotetext{
${ }^{3}$ Es lo que hace que nuestro proyecto tenga un valor, por ejemplo, capital humano, patentes, producto, estrategia de comercialización, entre otros.

${ }^{4}$ Los mercados no son perfectos, no todos los participantes en él tienen el mismo grado de información.

${ }^{5}$ Éstas pueden ser: inicio (start up), expansión, crecimiento, madurez y declive (Damodaran, 2002). En estas etapas el máximo crecimiento está antes de tener la madurez el proyecto.

${ }^{6}$ Tenemos el derecho, mas no la obligación de seguir con el proyecto.
} 
mas no la obligación, de seguir o no con el proyecto; los trabajos realizados por Black y Scholes (1973), Merton (1973), Cox, Ross y Rubinstein (1979) derivan la fórmula para la valoración de opciones para casos donde se conocen las distribuciones de probabilidad de eventos futuros.

Dixit y Pindyck (1994) utilizan la optimización dinámica para la toma de decisiones secuenciales en la vida del proyecto. Durante esa vida se presentan diferentes opciones de seguir o no con el proyecto. La relevancia de la valoración de proyectos consiste en verificar la viabilidad en la recuperación del capital invertido, además de un rendimiento asociado a un riesgo asumido. El método de valoración mediante el descuento de flujos de caja menos su inversión (valor presente neto, VPN) es el más aplicado, éste no toma en cuenta la volatilidad de las variables en el tiempo. En la vida del proyecto se presentan variables como costos, ventas, tasas de interés, entre otras, así como diversas opciones de decisión (flexibilidad). Este método presenta limitaciones en el momento de valorar el impacto de la incertidumbre de estas variables.

Otra alternativa relacionada con el VPN es el método de opciones reales (ver ecuación 1) que toma en cuenta las diversas opciones (flexibilidad) para poder alterar el curso de una acción planeada en el futuro, dada la información disponible en ese momento; existen distintas posibilidades como ampliar nuevos productos o mercados, de vender el proyecto, de abandonar el proyecto, de diferir el comienzo del mismo, entre otros.

donde

$$
V P N=V P_{\text {sin flexibilidad }}+V P_{\text {flexibilidad }}-I I
$$

$V P N$ - valor presente neto del proyecto

$V P_{\text {sin flexibilidad }}$ - es el valor presente del proyecto

$V P_{\text {flexibilidad }}$ - es el valor presente de las opciones que presenta el proyecto

II - es la inversión inicial

Si asumimos conocer los procesos estocásticos que siguen estas variables, el método alternativo para valorar esta flexibilidad es la simulación mediante el método Monte Carlo con regresiones de mínimos cuadrados (Longstaff, Schwartz, 2001); 
con éste es posible hacer simulaciones con diferentes tipos de opciones en el mismo proyecto (Gamba, 2002).

El riesgo prácticamente imposible de probabilizar nos lleva a suponer distribuciones de probabilidad (procesos estocásticos) para acontecimientos futuros, lo que deja a un lado posibles acontecimientos debido a que los forzamos a que estén dentro de una distribución de probabilidad, por lo que estaremos frente a problemas híbridos, donde tendremos información cierta (se conoce la distribución de probabilidad) e incierta (se desconoce la distribución de probabilidad), según sea el caso. Una relación de los métodos utilizados para la valoración de proyectos I+D y para la valoración de Knowledge-based enterprices (KBEs), se encuentra en Johnson, Neave y Pazderka (2002).

\section{Incertidumbre y selección de proyectos de I+D}

Existe dentro de los proyectos de I+D información incompleta e imprecisa; además, comprometer dinero o recursos dificulta la toma de decisiones. Los datos económicos y financieros exhiben dependencias que, frecuentemente, resultan de difícil modelización. Al final, todo depende de la habilidad para valorar el riesgo e incertidumbre frente a los beneficios y desventajas proyectados. Esta información desde el punto de vista de la teoría de los conjuntos borrosos ve una ambiguiedad o vaguedad entre la información y la falta de información. Cuando no se está seguro de la información se recurre a una forma probabilística, con la cual se tendrían dos puntos de vista: el borroso diría "la información es borrosa o incierta"; el probabilista señalaría "la información es inexacta o riesgosa" (Kosko, 1995).

Es necesario aclarar que a mayor precisión (grado) de la información, mayor borrosidad; en otras palabras, a mayor grado de precisión, mayor información; a mayor información mayor borrosidad; con más información se describen mejor los hechos. Por otra parte, la probabilidad es bivalente, un elemento puede pertenecer o no a un conjunto. Los conjuntos borrosos aparecen cuando un elemento pertenece parcialmente a un conjunto, pueden ser multivalentes, tienen cierto grado de pertenencia. Por ello, la probabilidad excluye información (dice si pertenece o no, excluye el grado de pertenencia), en cambio los conjuntos borrosos no porque tienen cierto grado de pertenencia. La lógica borrosa consiste en razonar con conjuntos borrosos; el conjunto borroso es la expresión de la información y la lógica borrosa consiste en el manejo de esta información. 
La lógica borrosa y las redes neuronales ${ }^{7}$ son herramientas que nos ayudan a comprender las imprecisiones, detalles pequeños y aspectos profundos de los diferentes hechos, sistemas, procesos y valores, políticas y objetivos. Asimismo, son herramientas que ayudan a predecir la interacción y desenvolvimiento de sucesos complejos para poder resolver problemas y visualizar otros todavía no conocidos. Esta determinación está captada por la teoría del caos. La teoría borrosa confirma esto, además menciona que todo es cuestión de grado (Kosko, 1995).

Innovar requiere crear algo nuevo, lo que trae consigo riesgos e incertidumbre ${ }^{8}$. Una organización innovadora para tomar decisiones requiere saber calcular y valorar los riesgos y la incertidumbre (Adair, 1992). Por ello, se insiste en el uso de la teoría de conjuntos borrosos para la selección de proyectos de I+D. Son cuatro los tipos de incertidumbre que se presentan (Ramírez, 1998), los cuales se exponen a la derecha de la figura 1 .

\footnotetext{
${ }^{7}$ Son sistemas de computación similares al cerebro, sirven para encontrar las reglas borrosas, las cuales tratan de ayudarnos a simular la realidad. Se busca un sistema que aprenda de la experiencia mediante reglas borrosas; es decir, aprender de los datos. Un sistema que sea capaz de convertir el comportamiento experto (juicio) en reglas borrosas.

${ }^{8}$ La predicción o la preocupación por el largo plazo no desaparece mediante la planificación, sino a través del aprendizaje complejo que se define por lo siguiente: 1) en el seguimiento de estrategias, 2) en la identificación de la cultura y 3) en la filosofía de la economía o en la organización; todo ello bajo la impredecibilidad inevitable del sistema propio de todo proceso innovador y creativo (Nieto, 1999).
} 


\section{Figura 1}

Tipos de incertidumbre

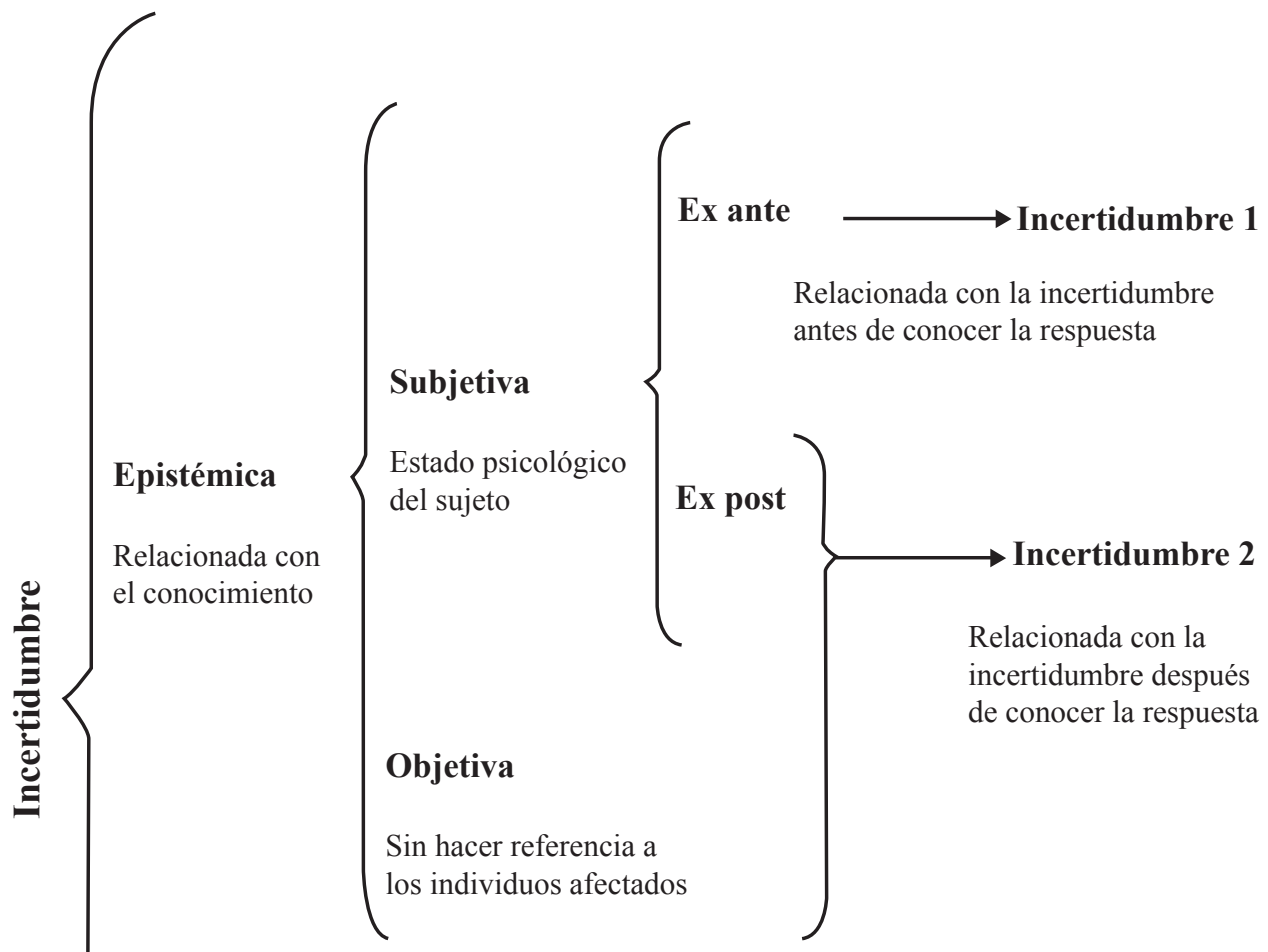

Ontica

Incertidumbre 3

Se relaciona con la incertidumbre sobre lo que es medible.

Volitiva

Incertidumbre 4

Aun teniendo el conocimiento hay duda, irresolución, puede estar relacionada con la moral.

Fuente: Ramírez (1998) y elaboración propia. 


\section{Avances en el uso de la teoría de conjuntos borrosos en la selección de pro- yectos de I+D}

Para la selección de las publicaciones se utilizaron las bases de datos del ISI Web of Knowledge ${ }^{9}$ (Social Science Citation Index y Science Citation Index), Science Direct y EBScohost (Busines Source Elite y Econlit). Se filtró con las palabras clave R\&D, Fuzzy y investment project entre 2001 a 2007. Se encontraron alrededor de 300 artículos que se seleccionaron por su relación con el tema y se agruparon por el tipo de problemática que se intentaba solucionar. Posteriormente, se seleccionó un artículo de cada grupo o subgrupo con el fin de que éste aportara algún diferencial en su metodología utilizada. Al final se obtuvieron diez artículos que proponían diferentes metodologías a problemas específicos, se tomó como base las metodologías mencionadas por Henriksen y Traynos (1999). El objetivo del estudio fue conocer la tendencia del conocimiento, problemáticas y metodologías relacionadas con la incertidumbre y la selección de proyectos de inversión; además, ubicar las fronteras del conocimiento en el tema. De igual forma, se trató de simplificar la búsqueda en los últimos años de soluciones a problemas específicos en la selección o valoración de proyectos de I+D. En el cuadro 1 se relacionan los diez artículos seleccionados y se presenta una breve explicación de los mismos.

Mohamed y McCowan (2001) propusieron un método para la selección de inversiones en el que aplicaron la fuzzy set theory y la qualitative possibility theory, en el manejo de la incertidumbre en aspectos monetarios y no monetarios de las opciones de inversión. Ordenaron los proyectos mediante un índice fuzzy que utiliza como insumos las diferencias de cada alternativa. Una de las bondades del método es la facilidad de cálculo y un fácil entendimiento del método.

Por su parte, Hsu, Tzeng y Shyu (2003) aplicaron fuzzy multiple criteria approach en la selección de proyectos de I+D en Taiwán. Este método incluye: 1) analytic hierarchy process (AHP), 2) un método de decisión grupal por expertos técnicos basado en los objetivos y criterios predeterminados, 3) la teoría de conjuntos borrosos para valorar la opinión de expertos. Sin embargo, tres características dificultaron la evaluación de proyectos del gobierno de Taiwán: considerar una diversidad de expertos, poco conocimiento sobre algunas tecnológica de ultima generación y desconocimiento de este tipo de tecnología relacionado con el riesgo

\footnotetext{
${ }^{9}$ Institute of Scientific Information (ISI).
} 
de mercado, falta de evaluadores y conocimiento técnico; es por ello que este tipo de evaluación tiende a ser subjetiva e intuitiva. Las ventajas del método fueron la solución de los aspectos señalados con base en el consenso sobre percepción de la evaluación y la de estimulación del valor de proyectos individuales; uno de los puntos por resolver consistió en que el modelo no consideró todas las posibilidades entre criterios y proyectos.

En tanto Carlsson y Fuller (2003) utilizaron la teoría de opciones donde el valor presente de las expectativas del flujo de efectivo y las expectativas de costos se representaron mediante números borrosos trapezoidales (fuzzy real option model). Se buscó determinar el tiempo óptimo para el ejercicio del proyecto, porque la opción de posponer una inversión tiene consecuencias de valor y de costo.

Por otro lado, Lin y Hsieh (2004) incorporaron los conceptos portfolio matrix model, fuzzy weighted average y fuzzy integer linear programming en la creacion de un Fuzzy Portfolio Selection System (FPSS), para integrar toda la información necesaria para la selección de proyectos y la administración de portafolios. Obtuvieron como resultado la reducción del tiempo en la toma de decisiones, manejo de la incertidumbre y un modelo flexible ante cambios en los parámetros de decisión.

A su vez Wang (2004) aplicó la teoría de conjuntos borrosos para desarrollar una metodología de asignación de tiempos en la realización de proyectos para el desarrollo de productos. Los parámetros de tiempo imprecisos (incertidumbre) se representaron con números borrosos. La medida qualitative possibility theory se utiliza para generar un algoritmo que mida los resultados en la asignación de tiempos y la forma como administradores adversos al riesgo toman decisiones. Esta metodología puede ayudar a los administradores en la colocación de recursos al minimizar el riesgo de iniciar tarde el desarrollo de productos para casos de proyectos que presenten incertidumbre en la información.

El modelo analytic hierarchy process (AHP) fue utilizado por Wang, Wang y $\mathrm{Hu}$ (2005) para la evaluación de proyectos I+D en diferentes disciplinas en China. El modelo, que fue estructurado al combinarlo con fuzzy scoring, se contrastó al ser utilizado por el ministerio de ciencia y tecnología de China. Tuvo como resultados la reducción del trabajo de expertos y gobierno, así como la transparencia del proceso. 
Choudhury, Shankar y Tiwari (2006) analizaron un problema de decisión grupal, en el que las variables por manejar eran del tipo multipersonal, de multicriterio y de multiescenarios de preferencias. Se utilizaron la transformación de algunas variables a relaciones de preferencia borrosa con el fin de eliminar al moderador de un grupo de decisión. El sistema se basó en dos criterios: en una medida del consenso que evalúa el consenso en cada situación y se emplea para guiar el proceso de decisión; y en una medida de proximidad que evalúa qué tan lejos está cada experto individualmente de la opinión colectiva, es utilizada como mecanismo de retroalimentación.

En cambio, Chiu, Chen, Shyu et al. (2006) tuvieron como objetivo la formulación y selección de la estrategia de lanzamiento de un nuevo producto. Cabe aclarar que la diferencia entre desarrollar productos de tecnología y tener productos innovadores es el éxito comercial de los productos innovadores. La clave está en el éxito, en la elección de las estrategias de lanzamiento del producto. Los autores utilizaron el método hierarchical fuzzy multicriteria decision-making (Fuzzy MCDM); además, presentaron su contrastación empírica en industrias de Taiwán.

Por su parte, Wang y Hwang (2007) desarrollaron un modelo para la selección de proyectos (en caso de existir información incierta) que ayudara en el armado de un portafolios de proyectos de I+D; esto contribuyó a maximizar los objetivos de valor y de contribución con la estrategia de la organización; para la valoración de los proyectos se utilizó fuzzy real option model. En este modelo la información para la toma de decisiones se presenta empleando conjuntos borrosos. Basado en la qualitative possibility theory se determina la solución óptima del modelo de selección de proyectos $\mathrm{I}+\mathrm{D}$ de tipo borroso. Esta teoría transforma el problema de programación linear del tipo borroso en un problema matemático de aversión al riesgo, el cual puede resolverse mediante una técnica de optimización.

Mientras tanto Wu y Lee (2007) analizaron cómo segmentar el tipo de competencias necesarias que debe tener un administrador para llevar con éxito una organización. Para resolver este problema, que involucra la vaguedad de los juicios humanos, estos autores emplearon un método que combinara fuzzy logic y Decision Making Trial and Evaluation Laboratory (DEMATEL) para segmentar los requerimientos de las competencias necesarias en los administradores.

El cuadro 1 resume el objetivo (o problemática) y la metodología de las publicaciones anteriores relacionadas con la selección de proyectos de I+D bajo incertidumbre. 


\section{Cuadro 1 \\ Relación de los artículos analizados \\ Enfoques basados en la teoría de los conjuntos borrosos en la selección de proyectos de I+D}

\begin{tabular}{|c|c|c|c|}
\hline Año & Autor y año & Objetivo & Metodología \\
\hline 2001 & $\begin{array}{l}\text { Mohamed y } \\
\text { McCowan }\end{array}$ & Selección de proyectos & $\begin{array}{l}\text { Fuzzy set theory, qualitative possibility } \\
\text { theory }\end{array}$ \\
\hline 2003 & Hsu, Tzeng y Shyu & Selección de proyectos & AHP y fuzzy scoring \\
\hline 2003 & Carlsson y Fuller & $\begin{array}{l}\text { Tiempo óptimo para iniciar el } \\
\text { proyecto }\end{array}$ & Fuzzy real option model \\
\hline 2004 & Lin y Hsieh & $\begin{array}{l}\text { Selección de proyectos y } \\
\text { armado de portafolios }\end{array}$ & $\begin{array}{l}\text { Portfolio matrix, fuzzy weighted average } \\
\text { y Fuzzy integer linear programming }\end{array}$ \\
\hline 2004 & Wang & $\begin{array}{l}\text { Asignación de recursos que } \\
\text { fomenten el inicio optimo en } \\
\text { el desarrollo de productos }\end{array}$ & $\begin{array}{l}\text { Fuzzy theory, qualitative possibility } \\
\text { theory }\end{array}$ \\
\hline 2005 & Wang, Wang y $\mathrm{Hu}$ & Selección de proyectos & AHP y fuzzy scoring \\
\hline 2006 & $\begin{array}{l}\text { Choudhury, } \\
\text { Shankar, Tiwari }\end{array}$ & Decisión grupal & $\begin{array}{l}\text { Fuzzy theory y similitudes con el método } \\
\text { Delphi }\end{array}$ \\
\hline 2006 & $\begin{array}{l}\text { Chiu, Chen, Shyu, } \\
\text { et al. }\end{array}$ & $\begin{array}{l}\text { Selección de estrategias para } \\
\text { el lanzamiento de un producto }\end{array}$ & Fuzzy MCDM \\
\hline 2007 & Wang y Hwang & $\begin{array}{l}\text { Selección de proyectos y } \\
\text { armado de portafolios }\end{array}$ & $\begin{array}{l}\text { Fuzzy real option model, qualitative } \\
\text { possibility theory }\end{array}$ \\
\hline 2007 & Wu y Lee & Selección de administradores & $\begin{array}{l}\text { Fuzzy logic, Decision Making Trial and } \\
\text { Evaluation Laboratory (DEMATEL) }\end{array}$ \\
\hline
\end{tabular}

En la figura 2 se observan los conceptos y metodologías encontradas en los artículos del cuadro 1. Éstos son un referente en la creación de un modelo de decisión en la selección y asignación de recursos a proyectos de I+D. De forma global se puede 
analizar el problema de selección en dos áreas: por una parte, decisión grupal donde se retroalimenta a los proyectos y se seleccionan; y, por otra, cómo administrar este portafolios de proyectos. Es muy importante tanto seleccionar proyectos como observar las relaciones de riesgo entre ellos y las proporciones en cómo se invierte; esta combinación puede aumentar el riesgo de la empresa, por ejemplo, invertir grandes montos en proyectos con una alta correlación. En la selección de proyectos se encontraron cuatro áreas de análisis: 1) la preselección de proyectos, donde las variables de entrada son los flujos de efectivo y las diferentes opciones que puede tomar el proyecto durante su vida sin considerar a las personas involucradas, el tiempo óptimo de inicio y la elección de estrategias de lanzamientos de productos o servicios, entre otros; 2) el tiempo óptimo para el lanzamiento del producto; 3 ) la elección de estrategias en el lanzamiento del producto, lo anterior puede llevar a que un producto o servicio se convierta en innovación; y 4) la selección de administradores o capital humano que actuará en el cumplimiento de objetivos. Los puntos anteriores se complementan para la selección de proyectos, además, pueden utilizarse en la mejora de las propuestas en proyectos de I+D.

\section{Figura 2}

\section{Relación de conceptos y metodologías de los artículos estudiados}

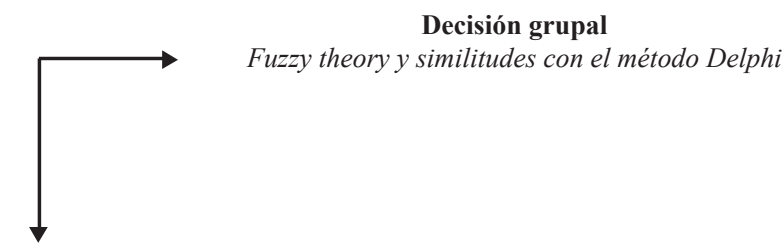

Armado de portafolios

Portfolio matrix, fuzzy weighted average y Fuzzy integer linear programming, Fuzzy real option model, qualitative possibility theory

1. Preselección de proyectos AHP, fuzzy scoring, Fuzzy real option model

2. Tiempo óptimo para el inicio del proyecto Fuzzy theory, qualitative possibility theory

3. Elección de estrategias de lanzamiento del producto Fuzzy MCDM

4. Selección de administradores Fuzzy logic, Decision Making Trial and Evaluation Laboratory (DEMATEL) 


\section{Conclusiones}

Los avances en el estudio en la selección de proyectos basados en la teoría de conjuntos borrosos han llevado a incorporar la incertidumbre en la valoración y selección. Las características de un producto innovador ${ }^{10}$ — por ejemplo, en su momento el sistema Windows, Google, entre otros- pueden confundir como suficiente condición el potencial de innovación que representan para su financiamiento, pero en la realidad muchas veces no está muy claro su éxito comercial, o su éxito en sus primeras etapas (investigación y desarrollo); su identificación es muy difícil.

En los documentos analizados se observan diferentes problemáticas por resolver; por un lado, en la selección; y, por otro, la mejora de una propuesta como la selección del tiempo óptimo para iniciar un proyecto y la selección de estrategias para el lanzamiento de un producto. En general, las bases metodologías analizadas caen dentro del análisis multicriterio, teoría de opciones y la teoría de la posibilidad, desde el punto de vista borroso (fuzzy).

Una de las aportaciones de este documento es la relación entre los conceptos y metodologías (ver figura 2) porque ayudan a formar un modelo de decisión en la asignación de recursos a proyectos de I+D y a indagar en cada área de análisis donde se presente incertidumbre. Como puede observarse en la misma figura no sólo se trata de seleccionar y retroalimentar, sino de mejorar la posición que se tenga en el armado del portafolios de proyectos I+D (relaciones de riesgo y rendimiento). Estas aportaciones servirán a investigadores y profesionales a facilitar la búsqueda de la frontera del conocimiento en relación con la incertidumbre en la selección y valoración de proyectos de I+D.

Aunque los diferentes trabajos presentan una estructura razonable para la solución de los diferentes problemas en la selección de proyectos, la forma de pensar y actuar de un inversionista en este tipo de proyectos puede ser diferente. El inversionista tiene en mente un conjunto de factores, por ejemplo, nivel de apalancamiento, costo de capital, crecimiento económico, entre otros, que le ayudan a tomar decisiones sobre la inversión en este tipo de empresas (empresas que invierten de I+D); estos factores tal vez no son los mismos que utiliza un administrador (problemas de agencia).

\footnotetext{
${ }^{10}$ Tecnometría.
} 
Conocer la óptima combinación de factores que utiliza el inversionista ayudará a mejorar la presentación de los proyectos a inversionistas y a aumentar las inversiones en este tipo de proyectos. Ho, Tjahjapranata y Yap (2006) intentan responder a este cuestionamiento, su modelo lineal explica sólo el 5\% de la varianza de los resultados de la empresa.

Para avanzar sobre estas líneas de investigación es necesario la búsqueda de factores que permiten la participación de inversionistas en proyectos de I+D y la selección y valoración de proyectos de I+D (aportaciones de este documento). Esto permitirá a inversionistas, a administradores de proyectos de $\mathrm{I}+\mathrm{D}$, a proveedores de créditos y a la política económica avanzar en la identificación de oportunidades de crecimiento económico mediante inversiones en I+D. Como se mencionó, este tipo de proyectos es un medio por el cual la innovación se extiende por la economía.

\section{Referencias}

Adair, J. (1992). El reto gerencial de la innovación. Santa Fe de Bogotá: Legis Editores.

Black, F. and Scholes, M. (1973). "Pricing of options and corporate liabilities". Journal of Political Economy, 81(3), 637-654.

Carlsson, C. and Fuller, R. (2003). "A fuzzy approach to real option valuation". Fuzzy Sets and Systems, 139(2), 297-312.

Chiu, Y. C., Chen, B. Shyu, J. Z. and Tzeng, G. H. (2006). "An evaluation model of new product launch strategy". Technovation, 26(11), 1244-1252.

Choudhury, A. K., Shankar, R. and Tiwari, M. K. (2006). "Consensus-based intelligent group decision-making model for the selection of advanced technology". Decision Support Systems, 42(3), 1776-1799.

Cox, J. C., Ross, S. A. and Rubinstein, M. (1979). "Option pricing-simplified approach”. Journal of Financial Economics, 7(3), 229-263. 
Damodaran, A. (2002). Investment valuation: Tools and techniques for determining the value of any asset (2nd ed.). New York: John Wiley \& Sons.

Dapena, J. P. (2003). "On the valuation of companies with growth opportunities". Journal of Applied Economics, 6(1), 49-72.

Dixit, A. K. and Pindyck, R. S. (1994). Investment under uncertainty. United Kingdom: Princeton University Press.

Escorsa, P. and Maspons, R. (2001). De la vigilancia tecnológica a la inteligencia competitiva. Madrid: Pearson Educación.

Fagerberg, J., Mowery, D. and Nelson, R. (2005). The oxford handbook of innovation. Oxford: Oxford University Press.

Gamba, A. (2002). Real Options Valuation: a Monte Carlo Simulation Approach, Working Paper: Faculty of Management, University of Calgary, 1-40.

Henriksen, A. and Traynor, A. J. (1999). "A practical R\&D project-selection scoring tool”. IEEE Transactions on Engineering Management, 46(2), 158170.

Ho, Y. K., Tjahjapranata, M. and Yap, C. M. (2006). "Size, leverage, concentration, and R\&D investment in generating growth opportunities". Journal of Business, 79(2), 851-876.

Hsu, Y. G., Tzeng, G. H. and Shyu, J. Z. (2003). "Fuzzy multiple criteria selection of government-sponsored frontier technology R\&D projects". $R \& D$ Management, 33(5), 539-551.

Jonson, L. D., Neave, E. H. and Pazderka, B. (2002). "Knowledge, innovation and share value". International Journal of Management Reviews, 4(2), 101-134.

Kosko, B. (1995). Pensamiento borroso: La nueva ciencia de la lógica borrosa. Barcelona: Drakontos.

Lin, C. H. and Hsieh, P. J. (2004). "A fuzzy decision support system for strategic portfolio management”. Decision Support Systems, 38(3), 383-398. 
Merton, R. C. (1973). "Theory of rational option pricing". Bell Journal of Economics, 4(1), 141-183.

Mohamed, S. and McCowan, A. K. (2001). "Modelling project investment decisions under uncertainty using possibility theory". International Journal of Project Management, 19(4), 231-241.

Nieto, U. (1999). Historia del tiempo en economía. Madrid: McGraw-Hill.

Ramírez, D. (1998). “Analysis of uncertainty”. Fuzzy Economic Review, 2(3), 69-80.

Wang, J. (2004). "A fuzzy robust scheduling approach for product development projects". European Journal of Operational Research, 152(1), 180-194.

Wang, J. T. and Hwang, W. L. (2007). "A fuzzy set approach for R\&D portfolio selection using a real options valuation model". Omega-International Journal of Management Science, 35(3), 247-257.

Wang, K. M., Wang, C. K. and Hu, C. (2005). "Analytic hierarchy process with fuzzy scoring in evaluating multidisciplinary R\&D projects in china". IEEE Transactions on Engineering Management, 52(1), 119-129.

Wu, W. W. and Lee, Y. T. (2007). "Developing global managers' competencies using the fuzzy DEMATEL method". Expert Systems with Applications, 32(2), 499-507. (A) 
